\title{
Multichannel excitation of the quasi-continuum
}

\author{
Ronald S. Burkey and C. D. Cantrell \\ Center for Quantum Electronics and Applications, University of Texas at Dallas, P.O. Box 688, Richardson, \\ Texas 75080
}

Received March 7, 1984; accepted June 18, 1984

\begin{abstract}
For a quantum system in which a continuous or quasi-continuous band of levels is excited by means of several dipole interaction channels, we develop a mathematical theory that simplifies the numerical solution of the Schrödinger equation. The theory allows time-varying electric fields (or electric-field envelopes). It also takes into account the exact shape of the band as well as the fact that different excitation channels see different band shapes. Accuracy of the results can be as high as desired. We show that the dephasing of the quasi-continuum probability amplitudes in the conventional continuum basis corresponds to the propagation of well-defined waves in the new basis that we introduce.
\end{abstract}

\section{INTRODUCTION}

Actual calculations involving real quantum systems can be difficult in the sense that so many levels interact in such a complicated way that calculations can require vast quantities of computer time. It is therefore common in quantum optics to construct simplified models in which one can easily study certain phenomena without necessarily trying to mimic any real system with the model. These phenomena include passage of optical pulses through media, intramolecular relaxation (IMR), and laser-induced dissociation.

One popular model is the $(1, N)$ system, in which a special state, the ground state, can interact with a band of $N$ levels but the $N$ levels cannot interact among themselves. These efforts are summarized by Shore. ${ }^{1}$ In this model, dense bands of levels absorb population from the ground state, making the model useful for the study of IMR. Another popular model is the ladder system, in which a series of states can interact with adjacent states but not with any other state. The first investigation employing the ladder-system model was that of Göppert-Mayer in 1931.2 In this model, multiphoton absorption can occur, making it possible (for example) to study questions of laser chemistry. Haydock ${ }^{3}$ showed that, in the case of a constant electric field (envelope), all discrete systems can be converted into ladder systems with reasonable ease. We have shown ${ }^{4}$ that the (1, band) system can be converted into a ladder system even if the band is continuous and the electric field varies and that this can be done with a similarity transformation independent of time. Thus there is a certain degree of unity in the two models.

However, these two models do not by themselves cover (even conceptually) all phenomena of interest. In particular, phenomenological damping is often added to the models. Lefebvre and Savolainen ${ }^{5}$ have developed a complicated model combining features of both $(1, N)$ systems and ladder systems, with many adjustable parameters. Witriol ${ }^{6}$ has also developed such models. Galbraith et al. ${ }^{7}$ generalized the treatment of $(1, N)$ systems in another way by coupling two $(1, N)$ systems [or, alternatively, by using a $(1, N)$ system in which each level is replaced by two degenerate levels]; they found that many of the results and methods previously applied to the $(1, N)$ case can be extended to cover this case as well.
Rather than a complex model, we present a mathematical theory that aids in the accurate reduction of models containing a continuous (or dense discrete) band of levels to a computable form. By this we mean that the Schrödinger equation is put into such a form that one can use a numerical differentialequation solver on a computer to determine the time evolution of the probability amplitudes. Our method of reduction has two basic virtues. First, time-varying electric fields are welcome. Second, continuous bands of levels may be present. For various reasons, some of which are discussed in Section 2 , the replacement of richly structured bands by phenomenological damping may not be entirely satisfactory. Therefore we not only take into account the exact band shape but also actually allow each level not in the band to perceive the band as having a different shape. (By the shape of the band we mean the functional form of the dipole operator matrix. Thus we do not force the dipole matrix into a specific form.)

Yeh et al. ${ }^{8}$ have considered another method for taking the band shape into account in their interrupted coarse-graining theory of $(1$, band $)$ systems. They easily derive qualitative information about the evolution of the system, given that the electric field is constrained to be slowly varying. On the other hand, our approach to the solution of $(N$, band) systems has no such constraint and was designed to produce highly accurate quantitative information.

We also present results of a numerical application of this technique. This example is interesting because some features of it are both analogous to and explainable by classical wave theory. Because of this, despite our comments in the above paragraph, our method can provide qualitative insight as well as quantitative information regarding the time evolution of the system.

All the approaches mentioned above, including our own approach, deal with the time evolution of the system as dictated by the Schrödinger equation for state vectors. Some phenomena, such as collisional damping, simply cannot be dealt with in this framework, requiring density-matrix equations of motion for adequate treatment. It may be possible to extend our method to cover this case, but, if so, we do not know how to do it at the present time. Therefore, although we state that the electric field may vary arbitrarily in 
time, it should be noted that the time of variation of the field (as well as the time interval of interest) is shorter than the collisional damping time.

\section{THEORY}

We present a method of dealing with continuous bands of levels, and we constantly refer to continuous bands throughout the paper. Actually, our results hold, to a large degree, for discrete bands (such as dense quasi-continuous bands). We speak this way, however, because the concepts that we use are more familiar in the continuous case and because we want to distinguish the band that we are simplifying from other (presumably discrete) levels in the system. Moreover, we speak of a classical electric field even though our results are good for a quantum field. With this in mind, consider a system consisting of $N$ discrete levels and a continuous band of levels. We allow dipole transitions among the discrete levels or between the discrete levels and the band; however, no dipole transitions are allowed within the band itself (see Fig. 1). Schematically, the Hamiltonian of a quantum system interacting semiclassically with an electric field can be written as

$$
H(t)=\delta+E(t) \mu .
$$

Our notation assumes that the rotating-wave approximation has been made, although this is by no means required in our approach. The operator $\delta$ is diagonal in the matrix representation that we use and contains the detunings of the levels; the form of the dipole matrix $\mu$ reflects the selection rules mentioned above. The electric-field envelope $E(t)$ is allowed to vary in time.

How should the band be treated computationally if we are to solve the Schrödinger equation numerically? The continuous band is often taken to act qualitatively as a reservoir, absorbing population until none remains in the discrete states. It is not uncommon to eliminate the band by introducing phenomenological damping in the form of complex detunings of the discrete states. ${ }^{6}$ This procedure is unsatisfactory in some ways, although the introduction of complex detunings is mathematically correct if the band has a Lorentzian shape. ${ }^{4}$ Although Lorentzian bands do sometimes arise, the Lorentzian band shape can by no means be thought of as typical because of the extremely slowly decreasing shoulders of the band. It is not clear if the Lorentzian-band approximation is reasonable quantitatively unless $a$ posteriori manipulation of the bandwidth (phenomenological damping rate) is allowed.

Consequently, we desire a more useful way of dealing with multichannel excitation of the continuum. Not only must any such method take the exact shape of the band into account in a more accurate way, but it also must not limit our ability to use a time-varying electric-field envelope $E(t)$.

Denote the detunings of the discrete levels by $\Delta_{k}$ (where $k=1, \ldots, N)$ and the detuning of a level in the band by $\Delta$ (where $\Delta_{\min } \leq \Delta \leq \Delta_{\max }$ ). Let $\mu_{j k}$ be the dipole matrix element connecting the $j$ th and $k$ th discrete levels, and let $\mu_{k}(\Delta)$ be the dipole matrix element connecting the $k$ th discrete level and the $\Delta$ th continuous level. In this basis, vectors and matrices are naturally partitioned into discrete and continuous parts. We will write our vectors, for instance, as having $N+$ 1 elements: The first $N$ elements are the actual elements of

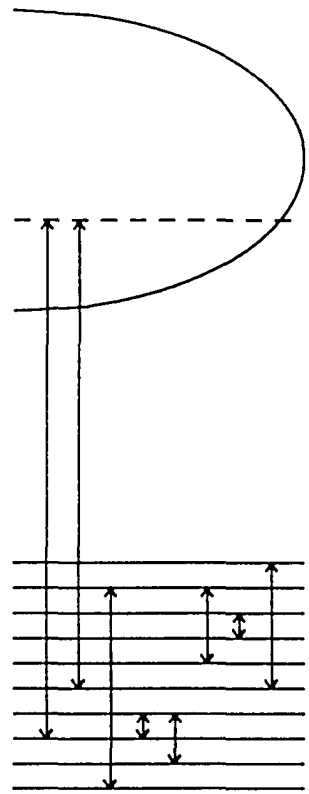

Fig. 1. A typical system in which a continuous band is excited through multiple channels. In general, the system may make any transition except those between two levels of the band.

the vector corresponding to discrete levels, and the last element combines all the elements corresponding to levels in the band in the compact form of a function of $\Delta$.

There is no reason to suppose that $\mu_{k}(\Delta)$, which represents the shape of the band as seen by the $k$ th discrete level, should be simply related to $\mu_{j}(\Delta)$ unless $j=k$. We will suppose, however, that there is a certain underlying shape $\mu(\Delta)$.of which all the $\mu_{k}(\Delta)$ are modifications. In particular, we assume that there are polynomials $p_{k}(\Delta)$ so that

$$
\mu_{k}(\Delta)=\mu(\Delta) p_{k}(\Delta) .
$$

By assumption, $\mu(\Delta)=0$ for $\Delta<\Delta_{\min }$ or for $\Delta>\Delta_{\max }$; thus $p_{k}(\Delta)$ need be accurate only in the interval $\Delta_{\min } \leq \Delta \leq \Delta_{\max }$. As an approximation, Eq. (1) can be made quite accurate, since by the Stone-Weierstrass theorem of real analysis theory, ${ }^{9}$ a continuous function can be approximated by polynomials on a closed finite interval to any desired degree of accuracy. Furthermore, the actual choice of $\mu(\Delta)$ is somewhat arbitrary. For a given level of accuracy in the approximation of Eq. (1), how closely $\mu(\Delta)$ approximates $\mu_{k}(\Delta)$ merely influences the degree of $p_{k}(\Delta)$ and not our ability to make (in theory) the approximation. That is, if $\mu(\Delta)$ is chosen poorly, then $p_{k}(\Delta)$ will be of higher degree.

It is possible to define a set of polynomials $f_{k}(\Delta)$, orthogonal with respect to the weight function $\mu(\Delta)^{2}$ (Refs. 10 and 11):

$$
\int_{\Delta_{\min }}^{\Delta_{\max }} \mu(\Delta)^{2} f_{k}(\Delta) f_{j}(\Delta) \mathrm{d} \Delta=\delta_{j k}
$$

Such orthogonal polynomials have proven quite useful in other investigations. ${ }^{3,4,12,13}$ We use them to define a new basis, in which the Hamiltonian will be conveniently simplified. Let our new basis be denoted by

$$
\mathbf{v}_{1}, \mathbf{v}_{2}, \ldots, \mathbf{v}_{N}, \mathbf{u}_{0}, \mathbf{u}_{1}, \ldots,
$$

where the $\mathbf{v}_{k}$ are the unmodified discrete states 


$$
\mathbf{v}_{1}=\left[\begin{array}{c}
1 \\
0 \\
\cdot \\
\cdot \\
\cdot \\
0 \\
0
\end{array}\right], \quad \mathbf{v}_{2}=\left[\begin{array}{c}
0 \\
1 \\
\cdot \\
\cdot \\
\cdot \\
0 \\
0
\end{array}\right], \ldots, \quad \mathbf{v}_{N}=\left[\begin{array}{c}
0 \\
0 \\
\cdot \\
\cdot \\
\dot{1} \\
0
\end{array}\right],
$$

$$
\begin{aligned}
\left\langle\mathbf{v}_{j}|\mu| \mathbf{v}_{k}\right\rangle & =\mu_{j k}, \\
\left\langle\mathbf{v}_{j}|\mu| \mathbf{u}_{k}\right\rangle & =p_{j k} \quad \text { if } k \leq M \\
& =0 \quad \text { if } k>M .
\end{aligned}
$$

Let us summarize what we have found so far. We have found a similarity transformation that is independent of time and of the field envelope $E(t)$ in which (1) the detuning matrix $\delta$ is tridiagonal, (2) the dipole matrix $\mu$ has only a few (i.e., a finite number) nonzero elements, and (3) all the matrix elements in the transformed system can be calculated easily. Put another way, in our basis the Hamiltonian is tridiagonal (and infinite) with a border that is not full [having only a finite number of nonzero elements, given by Eqs. (4a) and (6); see Fig. 2]. Since the transformation is independent of time, the Schrödinger equation can be solved (numerically, if desired) in the new basis. That is, for any quantum system containing a band of levels that do not interact among themselves, one can approximate uniformly the band shape to any desired degree of accuracy; furthermore, in the approximate system there is a similarity transformation that is independent of time for which the continuous band is converted into a ladder, of which only a finite number of the lowest levels are coupled to levels not in the ladder.

This result is a generalization of that for the $N=1, M=1$ case presented in Ref. 4 . It is also a special case of an even more general result that we will present in a future publication.

As an example, consider the case of two-channel excitation of the Tchebychev continuum

$$
\mu(\Delta)^{2}=\frac{2}{\pi \sigma}\left[1-\left(\frac{\Delta-s}{\sigma}\right)^{2}\right]^{1 / 2},
$$

where $s$ is the detuning of the center of the band and $\sigma$ is the half-width; i.e., $\Delta_{\min }=s-\sigma$ and $\Delta_{\max }=s+\sigma$. Chebychev's name is attached to this shape since the orthogonal polynomials formed are Chebychev polynomials of the second kind. Aside from the fact that, intuitively, this shape does not seem unreasonable, our reason for choosing it is that the elements of the tridiagonal Hamiltonian are extremely simple. ${ }^{14}$ Recall also that the choice of $\mu(\Delta)$ is arbitrary [if we are willing to accept the penalty of an increase in $M$ for a poor choice of $\mu(\Delta)$ ]. Thus, regardless of our motives for choosing this overall band shape, we are entitled to do so whatever the actual shape of the band. In Eqs. (4b) and (4c) we get

$$
1 / e_{n}=\sigma / 2
$$

and

$$
-d_{n} / e_{n}=s .
$$

For a numerical example, we set $\sigma=0.3 \mathrm{~cm}^{-1}, s=\Delta_{1}=\Delta_{2}=$ $\mu_{j k}=0$, and $p_{10}=p_{11}=p_{20}=1 / \sqrt{2}, p_{21}=-1 / \sqrt{2}$ (see Fig. $3)$. This represents two discrete levels that cannot interact with each other, one seeing the band as a modified Chebychev shape bunching up near $\Delta_{\max }$ and the other seeing the band bunching up near $\Delta_{\min }$. We initially put all the population in the ground state. The field is initially at zero but ramps linearly to $0.05 \mathrm{~cm}^{-1}$ at $t=1 / 30 \mathrm{nsec}$, remaining constant until $t=1 \mathrm{nsec}$ and then suddenly going to zero again. In the conventional continuum basis there are uncountably infinitely many coupled differential equations. . Therefore we work in the ladderlike basis of Fig. 2. Even here there are
Fig. 2. Hamiltonian matrix, in our basis, is infinite but discrete. It is tridiagonal with a border that is not full. The tridiagonal elements are independent of the electric field. 


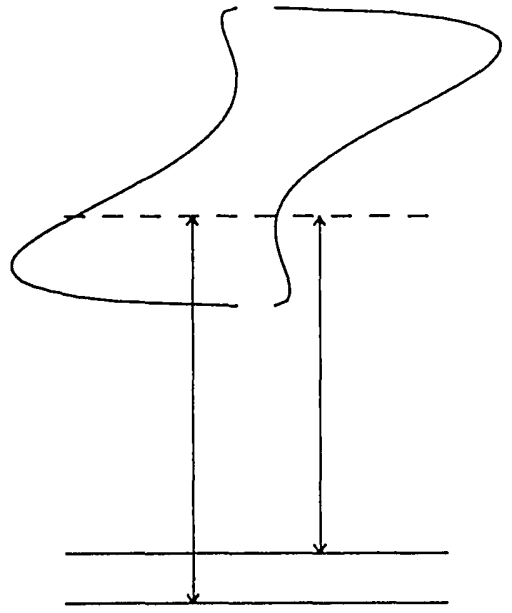

Fig. 3. A system in which a band of overall Chebychev shape is excited through two channels. The single continuous band is depicted twice to emphasize that the two discrete states see a different band shapes.

(countably) infinitely many levels. For the purpose of the example, we truncate the system and retain only the lowest 100 levels in the ladder. Thus, in practice, there are two approximations made; Eq. (1) approximates the band shape (allowing introduction of a discrete basis), whereas the truncation makes the number of levels finite. The Schrödinger equation is integrated from $t=0$. The results are shown in Fig. 4, in which population is plotted against level number; the
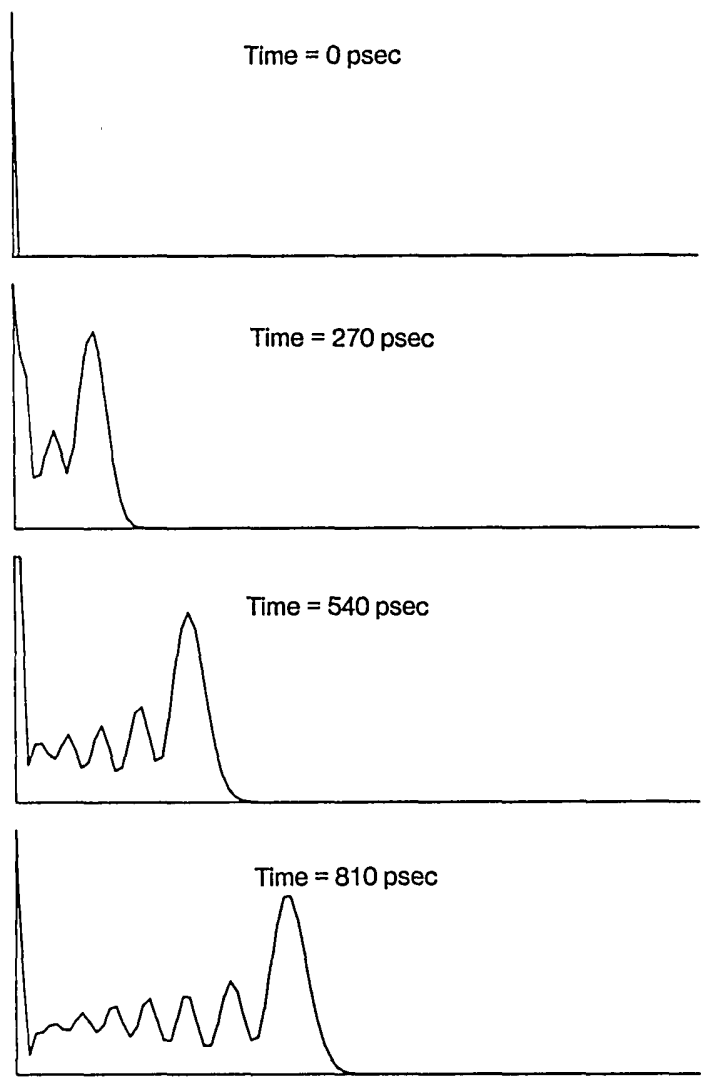

ground state is on the extreme left-hand side of the graphs, and the topmost level retained in the ladder is on the extreme right-hand side. We see an apparent pulse of population that forms near the discrete states and moves at a constant velocity up the ladder. When the pulse reaches the point at which we have truncated the ladder, however, it reflects (with a great deal of interference) and moves back toward the discrete states. Subsequent activity is shown in Fig. 5, which explores what happens when our approximation of the truncation of the band becomes important. When the reflected pulse reaches the discrete states, the field has been turned off, and there is no interaction, so the pulse is reflected again. In fact, it continues to be reflected for a (relatively) long time. If we had retained, say, only 50 levels in our ladder, Fig. 4 would be idential (to the eye) with our Fig. 4 until about $t=900 \mathrm{psec}$, at which time the pulse would reach the top of the ladder and begin to reflect. Basically, there is so little population in the highest levels (until the pulse reaches that area) that the number of levels retained in the truncated system is of no consequence almost until reflection begins. If this wavelike behavior could be counted on (we will show below that it can be, at least for the Chebychev band shape), and if we knew the speed of the pulses (which turns out to be $\sigma$ ), then we could easily determine the number of levels that must be kept in the ladder. If accurate probability amplitudes are desired for all levels, then the number of levels kept must be larger than the pulse speed multiplied by the time interval of interest. At the end of this time, however, the pulse reflects, and the truncation of the ladder becomes important. If, on the other hand,
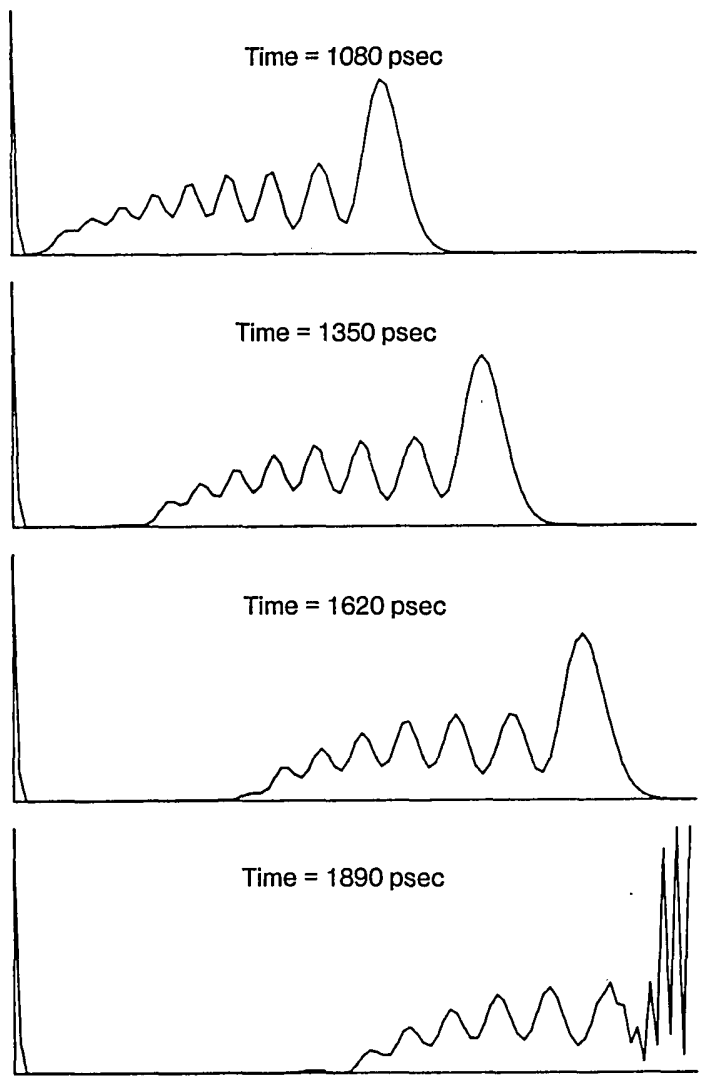

Fig. 4. Population versus level in the system of Fig. 3 in the ladder basis. The extreme left-hand level is the ground state, and the extreme right-hand level is the highest level kept in the ladder. The vertical scale runs from population $=0$ to population $=1 / 15$. (Any higher populations are truncated.) Apparently, a pulse of probability moves up the ladder. In the final frame ( $t=1890$ psec $)$, the interference pattern caused by the beginning reflection of the pulse is seen. 

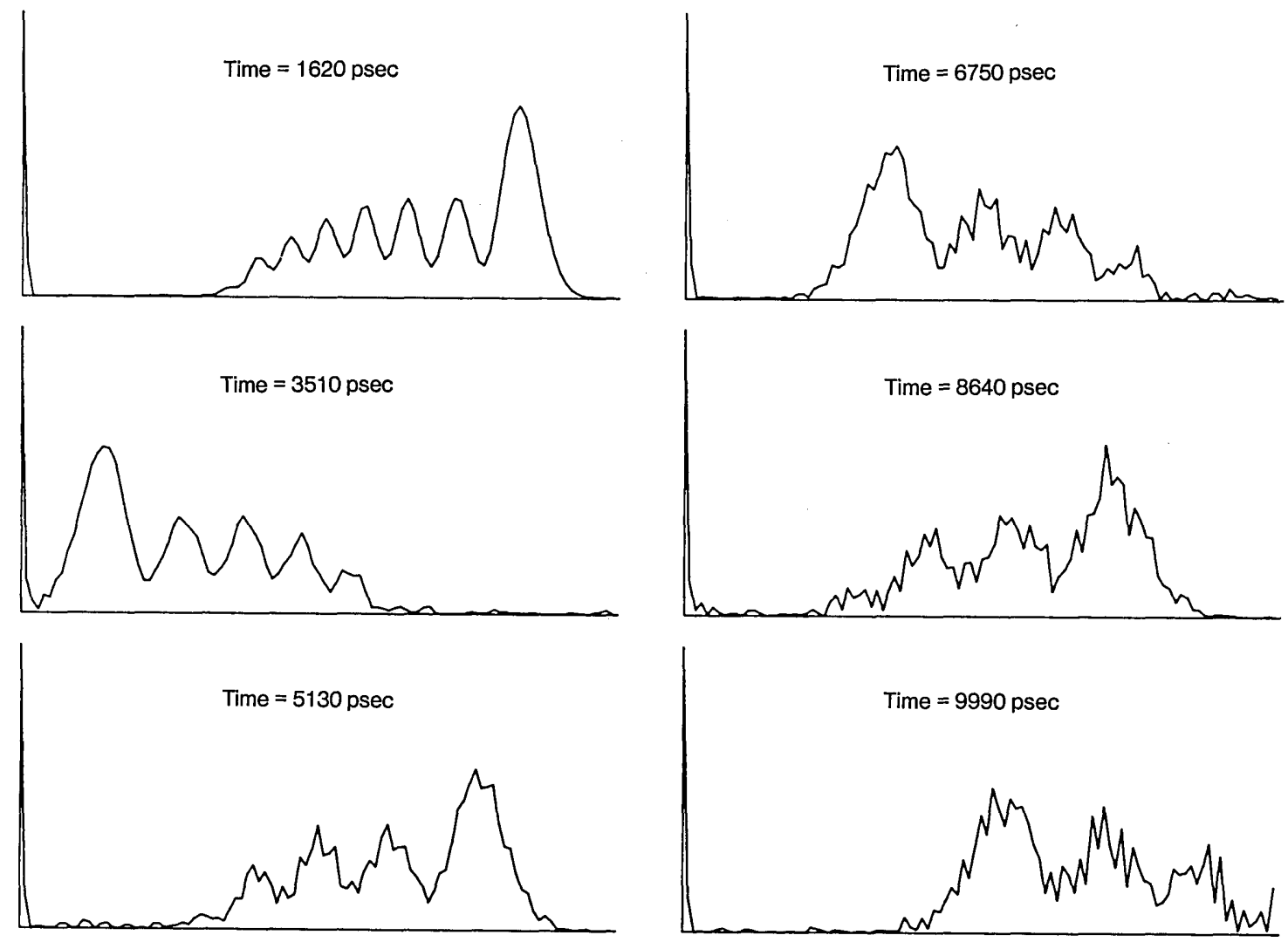

Fig. 5. Continuation of Fig. 4 for long times in which the truncation of the ladder makes all the band (ladder) populations inaccurate. The probability pulse continues to bounce around in the ladder, with one reflection occurring between successive frames.

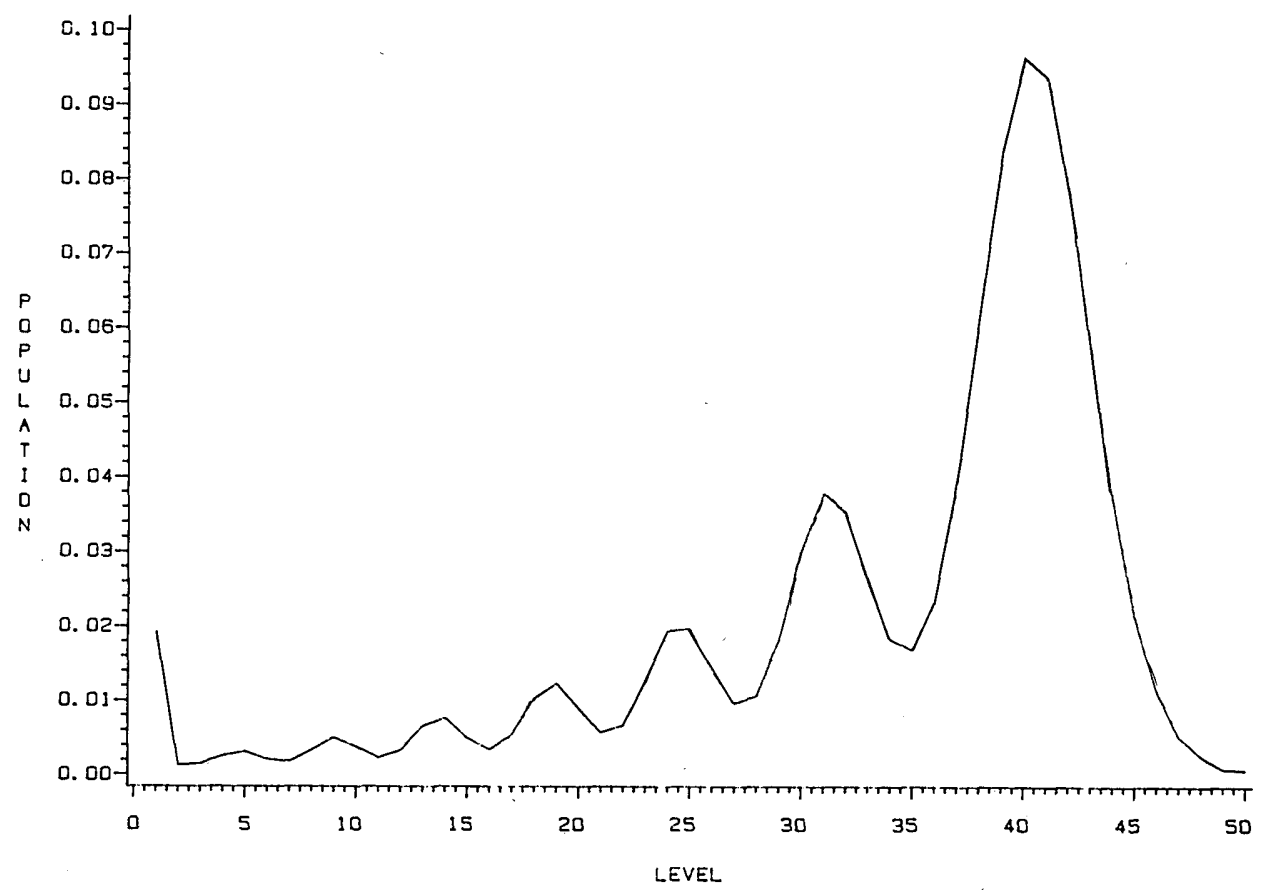

Fig. 6. Population versus level in a ladder basis, $N=1, M=1$ uniform rectangular band system. Time, 810 psec. The wave packet is moving to the right and has not yet reflected for the first time. HWHM of the band in the conventional continuum basis is $0.3 \mathrm{~cm}^{-1}$; the band center is at resonance; $\mu E(t) / 2 \hbar$ ramps linearly from zero to $0.05 \mathrm{~cm}^{-1}$ at $t=1 / 30 \mathrm{nsec}$, remaining constant thereafter.

we are interested in maintaining only the accuracy of the amplitudes of the discrete states (or of the complex polarization, ${ }^{4,13}$ which is almost the same thing), the requirements are less stringent. In this case, twice the number of retained levels must be less than the pulse speed multiplied by the length of time that the field remains on.

Although the Chebychev band shape was chosen for the simplicity of its tridiagonal detuning matrix, the effects seen 
above are not limited to the Chebychev band, as may be expected from the arbitrariness of $\mu(\Delta)$. For example, in Fig. 6 we see similar pulse propagation in the case of a uniform rectangular band with $N=1, M=1$. Nonetheless, the reason for this wavelike behavior is certainly easier to understand in the Chebychev case than in any other. Eberly et al. ${ }^{12}$ discovered these effects in a closely related system (a finite ladder with constant field) but concluded that a wave analogy was not entirely appropriate. For the Chebychev band, however, the wave analogy is instructive.

Consider an infinite Chebychev medium in which the quantities $a_{n}(n=0, \pm 1, \pm 2, \ldots)$, which can be viewed as probability amplitudes, obey the equation

$$
\mathrm{d} a_{n} / \mathrm{d} t=i\left[\left(\sigma / 2 a_{n-1}\right)+s a_{n}+\left(\sigma / 2 a_{n+1}\right)\right],
$$

which is recognizably the Schrödinger equation for those levels in the Chebychev ladder that are not directly coupled to any discrete states. As a trial solution, use

$$
a_{n}(t)=\exp [i(\omega t-k n)],
$$

which gives us the dispersion relation

$$
\omega=s+\sigma \cos k,
$$

from which follows an expression for the group velocity of wave packets ${ }^{15,16}$ :

$$
v_{g}=\partial \omega / \partial k=-\sigma \sin k .
$$

Slowly spreading wave packets will, of course, have the maximum or minimum values, namely, $v_{g}= \pm \sigma$. Except for unimportant phase factors [which can be removed by an appropriate transformation of $a_{n}(t)$ ], Eq. (7) is the dispersion relation of a classical, transversely vibrating, massless string mounted with massive beads. Another equivalent classical problem is that of longitudinally vibrating masses connected by springs. Thus it is no surprise that wave packets apparently move up and down the ladder at constant velocity. There is one difference from the two classical cases just mentioned in that the Schrödinger equation is a first-order differential equation, whereas wave equations are of second order. This means that, for a given $k$, there is no sign ambiguity in the group velocity. This is of little consequence unless the ladder system is truncated (the ends of the Chebychev medium are tied down), causing eventual reflections. However, it can be shown that the reflected wave is conjugated, thus reversing $k$ and the group velocity. In fact, it can be shown that the wave packets are distorted not by reflection but only by spreading. Thus, even if the electric field $E(t)$ is turned off, Chebychev wave packets can continue bouncing back and forth for a long time. Pulse spreading is exhibited in Fig. 5. The apparent cumulative degradation of the pulse is not due to the reflection of the pulse; rather, it is due to increasing interference with portions of the pulse that have spread but have not yet reflected. The motion need not represent any actual displacement of population in the band as seen in the conventional continuum basis; rather it represents an oscillation in the relative dephasing of the probability amplitudes in that basis. When the Chebychev pulse is near the top of the Chebychev ladder, the amplitudes are all dephased, and hence no process can extract population from the band; whether the field is on at this point is irrelevant. On the other hand, when the pulse is near the bottom of the ladder, the probability amplitudes of the band states in the original basis are nearly in phase, and hence the band can interact with the discrete states (if the field is turned on). Of course, if the field is turned off the populations in the band are always constant in the conventional continuum basis; regardless of changes in phase. The behavior of Chebychevlike waves could be considered to be the origin of recurrences in quantum systems.

Finally, we should make a remark about the choice of $\mu(\Delta)$ in practice. First, it is desirable to choose $\mu(\Delta)$ so as to minimize $M$ (thus simplifying interaction of the discrete states with the band). Second, it is desirable to choose $\mu(\Delta)$ so as to exhibit Chebychev waves (thus simplifying application of our qualitative knowledge). In fact, these considerations are not mutually exclusive. The most convenient overall band shape in practice is the weight function for Chebychev polynomials of the first kind. For this shape, only the first recursion coefficients $(n=0)$ in Eq. (3) differ from those discussed above, so Chebychev waves propagate just as before. On the other hand, it is well known that in expansion of function in terms of polynomials, use of Chebychev polynomials of the first kind generally produces the fastest convergence. That is, in general, this choice of $\mu(\Delta)$ minimizes $M$.

\section{SUMMARY}

We have discussed multichannel excitation of the quasicontinuum disguised as its inverse problem: multichannel excitation of a discretized form of a continuum. The system that we have considered has several discrete levels that may interact with one another semiclassically by means of a dipole interaction or with a separate continuous band of levels. We say that this is a disguise of the problem because most of the results that we have obtained hold equally well if the band is discrete. Treatments of similar problems sometimes insist that the electric-field envelope be constant or at least change slowly, but we have allowed the field to vary without restrictions on the rate of change or on the size of the field. Furthermore, we have taken into account the exact shape of the band and have even allowed the various discrete levels to perceive the band as being of different shapes.

Our general result is that there is a similarity transformation independent of the electric field (i.e., of time) that simplifies the Hamiltonian (and hence numerical solution of the Schrödinger equation) drastically. In this basis, the Hamiltonian is mostly tridiagonal, except for a finite number of matrix elements proportional to the electric field. Thus the continuous band turns into an (infinite) discrete ladder with only a few of the lowest-lying levels coupled to the original discrete levels by means of a dipole transition. Transitions within the ladder, however, are independent of the field.

We find that pulses of probability are injected into the ladder from the original discrete states. These pulses move up the ladder forever in the case of a real continuum, but if only a finite number of levels are retained (because of the necessity of performing computer calculations), the pulses eventually reflect and move down the ladder again. This reflection could be viewed as the origin of both error in discretization of continua and of recurrences in quantum systems. In the case of an overall Chebychev band shape, we proved that these pulses of population travel up and down the ladder at constant speed and experience distortion because 
of spreading but not because of reflections. The medium (i.e., the ladder) through which the wave packets move has the same dispersion relation as the classical, vibrating massless string on which are mounted massive beads. However, choice of the overall band shape is, to a large degree, arbitrary, so this effect is present in all systems with finite-width continuous bands.

\section{ACKNOWLEDGMENT}

This research is supported by Robert A. Welch Foundation under grant AT-873.

\section{REFERENCES}

1. B. W. Shore, "Coherence in the quasi-continuum model," Chem. Phys. Lett. 99, 240-243 (1983).

2. M. Göppert-Mayer, Ann. Phys. 9, 273 (1931).

3. R. Haydock, "The recursive solution of the Schrödinger equation," in Solid State Physics, E. Ehrenreich, F. Seitz, and D. Turnbull, eds. (Academic, New York, 1980), Vol. 35, p. 215.

4. R. S. Burkey and C. D. Cantrell, "Discretization in the quasicontinuum," J. Opt. Soc. Am. B 1, 169-175 (1984).

5. R. Lefebvre and J. Savolainen, "Memory functions and recurrences in intramolecular processes," J. Chem. Phys. 60, 2509-2055 (1974).

6. For example, N. M. Witriol, A. J. Galli, W. H. Brumage, and C. M. Bowden, "Criteria for the reduction of the effective manifold of states in models of laser-induced dissociation and chemistry," Opt. Lett. 5, 24-26 (1980); N. M. Witriol, "Including the continuum in the $N$-level molecule model," Chem. Phys. Lett. 98, 77-80 (1983).

7. H. W. Galbraith, J. R. Ackerhalt, and P. W. Milonni, "Coherent pumping in a weakly coupled quasi-continuum model," J. Chem. Phys. 78, 790-794 (1983).

8. J. J. Yeh, C. M. Bowden, and J. H. Eberly, "Interrupted coarsegrained theory of unimolecular relaxation and stimulated recurrences in photo-excitation of a quasi-continuum," J. Chem. Phys. 76, 5936-5946 (1982).

9. W. Rudin, Principles of Mathematical Analysis (McGraw-Hill, New York, 1964), Chap. 7.

10. D. Jackson, Fourier Series and Orthogonal Polynomials (Collegiate, Menasha, Wisc., 1941), Chap. 7.

11. R. Hamming, Numerical Methods for Scientists and Engineers (McGraw-Hill, New York, 1962), Chaps. 17-19.

12. J. H. Eberly, B. W. Shore, Z. Bialynicka-Birula, and I. Bialynicki-Birula, "Coherent dynamics of $N$-level atoms and molecules. I. Numerical experiments," Phys. Rev. A 16, 2038-2047. Also, Z. Bialynicka-Birula, I. Bialynicki-Birula, J. H. Eberly, and B. W. Shore, "Coherent dynamics of $N$-level atoms and molecules. II. Analytic solutions," Phys. Rev. A 16, 2048-2054.

13. C. D. Cantrell, V.S. Letokhov, and A. A. Makarov, in Coherent Nonlinear Optics: Recent Advances, M. S. Feld and V. S. Letokhov, eds. (Springer-Verlag, Berlin, 1980), Chap. 5.

14. U. Hochstrasser, in Handbook of Mathematical Functions, M. Abramowitz and I. A. Stegun, eds. (U.S. Government Printing Office, Washington, D.C., 1964), Chap. 22.

15. A. P. French, Vibrations and Waves (MIT Press, Cambridge, Mass., 1971), Chap. 7.

16. W. Pauli, Pauli Lectures on Physics (MIT Press, Cambridge, Mass., 1973), Vol. 2, Chap. 2. 